\title{
Pain in patients with transverse myelitis and its relationship to aquaporin 4 antibody status
}

\author{
Yazhuo Kong ${ }^{1,2}$, Helen Okoruwa ${ }^{2}$, Jon Revis ${ }^{2}$, George Tackley ${ }^{1,2}$, Maria \\ Isabel Leite ${ }^{2}$, Michael Lee ${ }^{1,2}$, Irene Tracey ${ }^{1,2}$, Jacqueline Palace ${ }^{2}$ \\ ${ }^{1}$ Oxford Centre for Functional Magnetic Resonance Imaging of the Brain \\ (FMRIB), University of Oxford, Oxford, UK \\ ${ }^{2}$ Nuffield Department of Clinical Neurosciences, University of Oxford, \\ Oxford, UK
}

\section{Corresponding author:}

Dr Jacqueline Palace,

Neuroscience Offices, L3, West Wing

Nuffield Department of Clinical Neuroscience

John Radcliffe Hospital

Headley Way

Oxford

OX3 9DU

Email: jacqueline.palace@ndcn.ox.ac.uk

Tel: +44 (0) 1865231900

Fax: +44 (0) 1865231870 


\section{Abstract}

Pain in transverse myelitis has been poorly studied. The aim of the study was to investigate the relationship between transverse myelitis related pain and disability, quality of life, anxiety and depression, cognitive-affective states in neuromyelitis optica (NMO) patients and aquaporin4 antibody status (AQP4$A b+v e$ as positive and AQP4-Ab - ve as negative). Transverse myelitis patients (44 in total; 29 AQP4-Ab +ve and 15 AQP4-Ab -ve) completed questionnaires including Pain Severity Index (PSI), Pain Catastrophising Scale (PCS), Hospital Anxiety and Depression Scale (HADS), Short Form-36 quality of life (SF-36 QOL). Clinical details such as disability, gender, age and spinal cord lesion type (short or long lesion) were noted. Correlation and multiple linear regression tests were performed using these clinical scores. Pain was found to be correlated strongly with quality of life in both groups but only correlated with disability in the AQP4-Ab +ve group. PCS, HADS and EDMUS were found to be highly correlated with pain severity using partial correlation, however, a stronger relationship between pain severity and PCS was found in the AQP4-Ab -ve group. Multiple regression analysis showed that pain severity was the most important factor for quality of life but not disability or anxiety and depression symptoms in the whole patient group. We confirm that pain is an important symptom of transverse myelitis and has more influence on quality of life than disability despite health services being predominantly focused on the latter. There may be different factors associated with pain between AQP4-Ab +ve and -ve patients.

Key Words: Neuropathic pain, quality of life, neuromyelitis optica, transverse myelitis, Aquaporin-4 antibodies 


\section{Introduction}

Transverse Myelitis (TM) is an acute inflammatory syndrome affecting the spinal cord that can be associated with significant disability [1]. There are many different diseases that can cause TM including multiple sclerosis (MS), para-infectious syndromes and neuromyelitis optica (NMO). NMO is a disease characterised by severe relapses of optic neuritis and longitudinally extensive transverse myelitis (LETM) and is associated with a pathogenic antibody to aquaporin 4 water channels (AQP4), the discovery of which has furthered our understanding of its pathogenesis as an astrocytopathy [2-6]. Despite chronic and resistant neurogenic pain being recently recognised to be major problem in NMO [7-11], the management of transverse myelitis currently focuses on reducing disability. The occurrence of pain in NMO patients with transverse myelitis was first highlighted by Kanamori et al. in 2011, who noted greater pain scores and worse quality of life scores in NMO compared to MS cohorts [7]. The pain tends to be distributed in the area of sensory involvement arising from the spinal cord lesion [7, 12]. The pathogenesis of pain is unclear but involvement of spinal cord grey matter (since the lesions are centrally located), brainstem descending modulatory pathways and astrocyte damage (due to autoantibodies targeting the AQP4 water channels located on astrocyte foot processes) are all likely contributors [13]. Central mechanisms and psychological factors are also recognised factors that contribute to a chronic pain phenotype in most other pain syndromes $[14,15]$.

In the current exploratory study, we have investigated the relationships between clinical factors (such as mood, disability, quality of life, and 
tendency to catastrophise), serum AQP4 antibodies (AQP4-Ab) and demographics (such as age) with pain intensity scores in patients who have suffered an attack of transverse myelitis seen within the national NMO service in Oxford. Because patients with AQP4-Ab have a specific pathogenesis, patients with and without this antibody were also assessed separately.

\section{Methods}

\subsection{Patients}

Myelitis patients seen within our NMO service are atypical (usually have longer lesions and more severe attacks) and include patients with the NMO antibody (AQP4-Ab) and those without. Patients with the more common typical MS TM or clinically isolated syndromes would not have been included as they are seen in MS clinics. All patients (82 in total) seen within the Oxford National NMO service in 2013 with an attack of myelitis, outside of relapse and in remission, were suitable for inclusion. The definition of a TM attack was an acute neurological attack, anatomically attributable to a lesion in the spinal cord, shown on MRI, and consistent with an inflammatory cause, including sensory symptoms \pm motor \pm bladder involvement.

Consecutive TM patients were asked to fill in questionnaires assessing pain, , anxiety and depression, quality of life and pain catastrophising scores. $50 \%$ patients returned fully completed forms in all areas and only data from these patients were analysed. 
Data from these questionnaires was collated with their gender, age, physical disability, an ambulation related score as a surrogate for spinal cord tissue damage, lesion type (LETM or short lesion) and length, number of relapses that was associated with the onset of pain, and their serum AQP4-Ab status. Physical disability, routinely collected from clinic visits, was assessed by the European Database for Multiple Sclerosis Scores (EDMUS) [16], a whole integer disability score which equates to the whole integer scores of the Expanded Disability Status Scale (EDSS). Five patients with significant visual impairment (at least one eye with poor vision; visual acuity on Snellen chart $\leq 6 / 36$, and the other moderate or worse; visual acuity on Snellen chart $\leq 6 / 18$ ) were highlighted because this might independently affect their quality of life and mood scores. Patients were asked to score their pain outside of pain associated with optic neuritis (ON).

Data from 43 patients (Table 1) with myelitis: 29 AQP4-Ab +ve and 13 AQP4Ab -ve were obtained for this analysis. The antibody negative group consisted of monophasic LETM (n=8, of whom 3 were myelinoligodendrocyte glycoprotein antibody positive), one NMO patient, and despite being referred as NMO patients we concluded 4 probably had MS (3 with an optico-spinal phenotype and one with a short TM), and one further patient had a monophasic short TM without any other identifiable cause.

\subsection{Standard protocol approvals}

The use of our patient data is covered by UK IRAS ethics approval number 10/H0606/56, and all participants provided written informed consent. 


\subsection{Questionnaires}

These self-reporting questionnaires were included: Pain severity index (PSI), Hospital Anxiety and Depression Scale (HADS), Pain Catastrophising Scale (PCS), Short Form-36 (SF-36) for quality of life (QOL). These questionnaires have been used extensively in studies of pain [17-20].

2.3.1 Pain severity index (PSI). The BPI-short form (brief pain inventory) measures pain severity (four questions) and the functional impact of pain on daily functions (seven questions) including location of pain, pain medications and amount of pain relief in the past 24 hours or the past [21] [22]. Each question is scored subjectively on a scale of 0 to 10,0 meaning no pain and 10 representing the worst pain possible[7]. Mild pain is defined as a worst pain score of $1 \sim 4$, moderate pain is defined as a worst pain score of $5 \sim 6$, and severe pain is defined as a worst pain score of 7 10 [21]. Combined all four pain severity scores in the questionnaire, 28 was used as severe pain threshold in the post-hoc analysis. We selected the pain severity index as a measure of pain severity for analysis.

2.3.2 Pain catastrophising scale (PCS). The current version was used in our study, developed and validated by Sullivan and colleagues [23]. The PCS measures pain intensity, emotional distress, pain-related disability, and pain behaviour. PCS was used to consider whether thoughts and feelings towards pain, are influencing the patient's perception of their pain experience. PCS is comprised of 12 questions split into three sections. It measures to what extent the patient focuses on their pain ('rumination') the extent to which the 
patient magnifies their pain experience ('magnification') and if the patient feels helpless because of their pain ('helplessness') [24]. The results of each question were converted to a scale ranging from 0 being the lowest level of pain catastrophising, to 4 being the highest level of pain catastrophising [24]. The higher the overall score for each of the three sections, the higher the level of pain catastrophising. We used the overall score of all three sections as total PCS measure.

2.3.3 Hospital anxiety and depression scale (HADS). The HADS score [25] [26]was used to examine the psychological or mental wellbeing of the NMO patients. The questionnaire consists of seven questions to identify anxiety and seven questions to identify depression. Grading for severity of the condition, was considered as scores of $0-7,8-10,11-14$ and 15-21, defining normal, mild, moderate and severe, respectively [27, 28].

2.3.4 SF-36. The SF-36 [29] [30] questionnaire is a set of 36 questions used to measure quality of life across different disease populations. The questionnaire covers physical functioning (10 questions), role limitations due to physical health (4 questions), role limitations due to emotional problems (3 questions), energy or fatigue (5 questions), emotional well-being (5 questions), social functioning (2 questions), pain (2 questions)) and general health (5 questions) [7]. Each question is graded from zero (worst) to 100 (best). We used the overall score of all 36 questions as the total measure of quality of life.

\subsection{Outcomes and statistical analysis}


The data was tested for normality and we found that EDMUS $(p=0.001)$ and PCS $(p=0.001)$ did not pass the Kolmogorov-Smirnov test. Therefore, in this study we applied non-parametric analysis for group comparisons and correlations. The Mann-Whitney test was used to explore if there were significant differences between the two AQP4-Ab groups (positive and negative) for EDMUS, PSI, HADS, PCS, SF36 and age.

Many of the measures are inter-dependant thus in order to explore the relationship between pain and individual factors (age, AQP4 status, lesion type and length, number of relapses, EDMUS score, and all the questionnaire scores), spearman correlations were performed without correction for multiple comparisons. Because studies have shown that visual impairment is linked to worse quality of life [31]we felt blindness might be a confounder. Thus patients with severe visual loss (defined as a visual acuity of $6 / 36$ in one or both eyes due to optic neuritis, ON) were noted when exploring the correlation between pain and quality of life. We were interested in factors that might contribute to pain severity, thus from the factors that correlated with pain individually and that were likely to have a causative role (PCS, HADS, EDMUS) we performed partial correlations adjusting for other likely confounding factors (age, gender and lesion type).

As there is a strong correlation between pain score and quality of life, we planned to explore how important pain was for quality of life compared to other factors. A multiple linear regression model was used to determine the most significant independent predictors of quality of life using the SF36 score as the dependant variable, with those factors that strongly correlated individually with the SF36 i.e. pain (PSI), HADS, age, PCS and disability 
(EDMUS) as independent variables. NMO is a female predominant disorder. Gender was not included in the regression analysis because there were only 8 males included the study. The residuals of the regression model were found to be normally distributed.

The partial correlations and the multiple linear regression analysis were calculated for the whole TM group. Because the AQP4-Ab positive group have a single distinct pathogenic mechanism and the AQP4-Ab negative group are heterogeneous, analysis was also performed for two separate groups. Due to the recognition of the relatively small sample size (the rarity of the condition) power analysis for this linear regression model was performed and demonstrated that with a total sample size of 43 and 5 independent variables would achieve a reasonable effect size $\left(f^{2}=0.35\right)$ and power (1$\beta=0.8)$. Subgroup analysis of the AQP4 positive and negative groups was performed for exploratory purposes because the study was not powered to do this.

Statistical analyses were performed using SPSS Mac version 21 (SPSS Inc). All statistical tests were 2-sided and $p<0.05$ was considered statistically significant.

\section{Results}

Table 1 summarises the clinical characteristics and the questionnaire scores of the patients which are grouped according to their AQP4-Ab status. Although there were no significant differences between the two groups for EDMUS, PSI, HADS, PCS, SF36 and age (Mann-Whitney, Table 1), severe 
pain severity scores $(\geq 28)$ were seen in more AQP4-Ab positive patients (34\%) than those without antibodies (13\%). Seventeen patients (13 AQP4-Ab positive and 4 AQP4-Ab negative) were taking anti-neuropathic medications (Gabapetin, Pregabalin, Carbamazepine, Oxcarbazepine or Amytriptiline); 11 patients were taking over the counter analgesics (6 AQP4-Ab positive and 5 AQP4-Ab negative) such as Paracetamol, Ibuprofen and Codeine; 9 patients (all AQP4-Ab positive) were taking anti-spasmodics including Duloxetine, Baclofen, Diazpam and Clonazepam.

[Table 1] here

The direct correlations (shown in sup Table s1 3) were performed and many of the variables were found to be correlated as expected. The correlation of QOL with pain severity was high in the whole group and in the subgroups. Bilateral visual impairment from optic neuritis (ON) was present in four AQP4-Ab +ve patients however their presence did not appear to affect the association between pain and QOL (Figure 1). Not surprisingly QOL and HADS, QOL and PCS, and HADS and PCS were all directly correlated. Of interest, pain severity was significantly correlated with disability in the AQP4Ab positive group but not the AQP4-Ab negative group, and HADS was significantly correlated with pain severity in the AQP4-Ab negative group but not the AQP4-Ab positive group. The pain severity did not correlate with number of relapses nor length of spine lesions in the whole group nor in the AQP4-Ab positive and negative patient groups.

[Figure 1] here

Additional factors that correlated with pain in the whole group were EDMUS, HADS and PCS therefore these were checked further by the partial 
correlation analysis. The partial correlations between pain severity and PCS, HADS and EDMUS were still significant for whole patient group after controlling for gender, age, and lesion type (Table 2). However, correlations between PSI and PCS were significantly different for antibody positive and negative groups $(p=0.05$, Table 2$)$ with a stronger relationship in the antibody negative group.

\section{[Table 2] here}

Factors that were significantly correlated with quality of life in the whole group (PCI, EDMUS, HADS, PCS, gender and age) were used in the multiple regression analysis. The multiple linear regression analysis using quality of life as the dependant variable (Table 3 ), showed that for the whole group, pain severity followed by age were significant factors. When dividing the groups into AQP4-Ab positive and negative groups, pain severity $(p=0.00035)$, disability score EDMUS $(p=0.015)$ and HADS $(p=0.018)$ were significant predictors in AQP4-Ab +ve patients; whereas in AQP4-Ab -ve patients only age $(p=0.024)$ was a significant predictor.

\section{[Table 3] here}

\section{Discussion}

Pain in transverse myelitis has been poorly studied. This study has highlighted that pain is an important symptom in patients with previous attacks of transverse myelitis seen in an NMO clinic. Pain was also correlated with anxiety and depression, disability and pain catastrophizing behaviour and the latter appeared more dominant in AQP4-Ab negative patients. Pain rather than disability and anxiety and depression was related to quality of life in the multiple regression analysis of the whole group. 
A previous study has shown that $46 \%$ of Japanese NMO and NMOSD patients [7] had moderate or severe pain severity scores and $22 \%$ had severe pain [7]. This is lower than the $58 \%$ and $34 \%$ in our UK AQP4-Ab NMOSD cohort and may be explained by the previous cohort not having an absolute requirement for AQP4-Ab positivity. Additionally not all our patients filled in the questionnaires and there may have been a positive selection bias i.e. those with more pain taking the trouble to fill in questionnaires. However our figures are similar to those of one study who reported neuropathic pain in $62 \%$ of UK patients (the majority being AQP4-Ab positive) [9]. Of note we have previously reported less disability in Japanese versus UK AQP4-Ab positive patients [32] and it is possible that genetic or cultural factors (either in pain interpretation itself or in pain pathology) might explain differences between these ethnic groups.

As in a previous study [9], we found no significant difference in the absolute pain scores, disability and quality of life between AQP4-Ab positive and negative groups. However, we explored further the relationship between pain severity and other factors. Our results did suggest that pain catastophising behaviour had a stronger effect in antibody negative patients. The correlation of pain with anxiety and depression is not surprising, and with disability suggests that the pathological damage of the transverse myelitis is an important factor in pain pathways.

There were other differences in the relationships we studied between AQP4$\mathrm{Ab}$ positive and negative groups which were interesting. Individual unadjusted pain correlations identified disability as significant factor only in the AQP4-Ab positive group and anxiety and depression only in the AQP4- 
Ab negative group. With the significant difference in the association with catastrophising behaviour this might suggest pain is influenced more by spinal cord pathology in AQP4-Ab positive patients and more by mood and personality in AQP4-Ab negative patients. However the small number of AQP4-Ab negative patients may reduce the power to detect an effect and the differences observed need confirming in further studies.

Severe bilateral visual impairment was found only in AQP4-Ab +ve patients although the numbers were small. The pain scores from visually impaired AQP4-Ab positive patients appeared to correlate similarly with $\mathrm{QOL}$ than those without visual impairment. It is noteworthy that disability (mobility led and visual) appeared to associate with QOL less than pain. This is understandable because patients can adapt to physical impairment more easily than uncontrolled pain. However health service resources tend to focus more on the disability than the pain.

The limitations of our observations include that only half our patients filled in all their questionnaires and a selection bias may have led to patients with more severe pain completing the scores. However this bias would have existed in both patient groups and although it could have affected the absolute scores it is unlikely to explain the relationships between the scores and there were a wide range of pain scores in both groups. Additionally, our small sample size may limit the power to see relationships with interdependence in multiple regression analysis and our multiple comparisons were not Bonferroni corrected. This was however an exploratory study to generate future hypothesis generated projects. Finally, only a general pain score rather than a neurogenic pain scale was used, thus 
other causes of pain could be confounders. However patients were asked to score their myelitis pain (which is usually quite different from other pains) and only two of our patients (one AQP4-Ab positive and one negative) had other causes of ongoing pain i.e. arthralgia due to osteoarthritis. Removing these patients does not alter the main results.

In summary our findings suggest that pain is an important factor in the quality of life experienced by both AQP4-Ab positive and negative transverse myelitis patients and suggest an even greater association than disability. A comprehensive effort to manage pain more effectively in these patients is supported. The association of an ambulation disability score (a reasonable surrogate for spinal cord tissue damage) with pain severity in this AQP4-Ab positive group supports our hypothesis that the AQP4-Ab tissue damage in the spinal cord (rather than lesion length) may be important in the pathogenesis of the resulting pain. . In contrast pain and quality of life in AQP4-Ab negative patients may be more complex and involve other factors that are less directly related to the disease pathology such as pain perception and behaviour. Thus pain management strategies in transverse myelitis patients may differ dependant on their AQP4-Ab status and this preliminary data supports the role for further research into transverse myelitis pain.

\section{Disclosure of conflict of interest}

None. 


\section{Acknowledgement}

This work was performed within the UK NMO service that is funded by the National Health Service (NHS). The authors report no disclosures relevant to the manuscript.

\section{Reference}

[1] West TW. Transverse myelitis--a review of the presentation, diagnosis, and initial management. Discovery medicine. 2013;16:167-77.

[2] Wingerchuk DM, Lennon VA, Pittock SJ, Lucchinetti CF, Weinshenker BG. Revised diagnostic criteria for neuromyelitis optica. Neurology. 2006;66:14859.

[3] Fujihara K. Neuromyelitis optica and astrocytic damage in its pathogenesis. Journal of the neurological sciences. 2011;306:183-7.

[4] Palace J, Leite MI, Jacob A. A practical guide to the treatment of neuromyelitis optica. Practical neurology. 2012;12:209-14.

[5] Melamed E, Levy M, Waters PJ, Sato DK, Bennett JL, John GR, et al. Update on biomarkers in neuromyelitis optica. Neurol Neuroimmunol Neuroinflamm. 2015;2:e134.

[6] Wingerchuk DM, Banwell B, Bennett JL, Cabre P, Carroll W, Chitnis T, et al. International consensus diagnostic criteria for neuromyelitis optica spectrum disorders. Neurology. 2015;85:177-89.

[7] Kanamori Y, Nakashima I, Takai Y, Nishiyama S, Kuroda H, Takahashi T, et al. Pain in neuromyelitis optica and its effect on quality of life: a crosssectional study. Neurology. 2011;77:652-8. 
[8] Qian P, Lancia S, Alvarez E, Klawiter EC, Cross AH, Naismith RT. Association of neuromyelitis optica with severe and intractable pain. Archives of neurology. 2012;69:1482-7.

[9] Zhao S, Mutch K, Elsone L, Nurmikko T, Jacob A. Neuropathic pain in neuromyelitis optica affects activities of daily living and quality of life. Multiple sclerosis. 2014.

[10] Carnero Contentti E, Leguizamon F, Hryb JP, Celso J, Pace JL, Ferrari J, et al. Neuromyelitis optica: association with paroxysmal painful tonic spasms. Neurologia. 2015.

[11] Bradl M, Kanamori Y, Nakashima I, Misu T, Fujihara K, Lassmann H, et al. Pain in neuromyelitis optica-prevalence, pathogenesis and therapy. Nature reviews Neurology. 2014.

[12] Leonard H.A.C. RJ, Matthews L.A.E., Kitley J., Leite M.I. , Palace J. Pain in neuromyelitis optica spectrum disease: a cross-sectional study in an English cohort. 28th ECTRIMS, Devic's NMO spectrum. 2012;October 12, 2012.

[13] Ji RR, Berta T, Nedergaard M. Glia and pain: is chronic pain a gliopathy? Pain. 2013;154 Suppl 1:S10-28.

[14] Price DD. Psychological and neural mechanisms of the affective dimension of pain. Science. 2000;288:1769-72.

[15] Scholz J, Woolf CJ. Can we conquer pain? Nature neuroscience. 2002;5 Suppl:1062-7.

[16] Amato MP, Grimaud J, Achiti I, Bartolozzi ML, Adeleine P, Hartung HP, et al. European validation of a standardized clinical description of multiple sclerosis. Journal of neurology. 2004;251:1472-80. 
[17] Fossa SD, Dahl AA. Short Form 36 and Hospital Anxiety and Depression Scale. A comparison based on patients with testicular cancer. Journal of psychosomatic research. 2002;52:79-87.

[18] Jensen MP, Ehde DM, Hoffman AJ, Patterson DR, Czerniecki JM, Robinson LR. Cognitions, coping and social environment predict adjustment to phantom limb pain. Pain. 2002;95:133-42.

[19] Lame IE, Peters ML, Vlaeyen JW, Kleef M, Patijn J. Quality of life in chronic pain is more associated with beliefs about pain, than with pain intensity. European journal of pain. 2005;9:15-24.

[20] Arola HM, Nicholls E, Mallen C, Thomas E. Self-reported pain interference and symptoms of anxiety and depression in community-dwelling older adults: can a temporal relationship be determined? European journal of pain. 2010;14:966-71.

[21] Cleeland CS, Ryan KM. Pain assessment: global use of the Brief Pain Inventory. Ann Acad Med Singapore. 1994;23:129-38.

[22] Cleeland C. Research in cancer pain. What we know and what we need to know. Cancer. 1991;67:823-7.

[23] Sullivan MJL, Bishop SR, Pivik J. The Pain Catastrophizing Scale:

Development and validation. Psychol Assessment. 1995;7:524-32.

[24] Chibnall JT, Tait RC. Confirmatory factor analysis of the Pain

Catastrophizing Scale in African American and Caucasian Workers'

Compensation claimants with low back injuries. Pain. 2005;113:369-75.

[25] Zigmond AS, Snaith RP. The hospital anxiety and depression scale. Acta Psychiatr Scand. 1983;67:361-70. 
[26] Aylard PR, Gooding JH, McKenna PJ, Snaith RP. A validation study of three anxiety and depression self-assessment scales. Journal of psychosomatic research. 1987;31:261-8.

[27] Vignaroli E, Pace EA, Willey J, Palmer JL, Zhang T, Bruera E. The Edmonton Symptom Assessment System as a screening tool for depression and anxiety. Journal of palliative medicine. 2006;9:296-303.

[28] Smart KM, Blake C, Staines A, Doody C. Self-reported pain severity, quality of life, disability, anxiety and depression in patients classified with 'nociceptive', 'peripheral neuropathic' and 'central sensitisation' pain. The discriminant validity of mechanisms-based classifications of low back (+/-leg) pain. Manual therapy. 2012;17:119-25.

[29] Jenkinson C. Quality of life measurement: does it have a place in routine clinical assessment? Journal of psychosomatic research. 1994;38:377-81. [30] Turner-Bowker DM BP, Ware JE., Jr. SF-36® Health Survey \& "SF" Bibliography: Third Edition (1988-2000) Lincoln, RI: QualityMetric Incorporated. 2002.

[31] Stelmack J. Quality of life of low-vision patients and outcomes of lowvision rehabilitation. Optom Vis Sci. 2001;78:335-42.

[32] Kitley J, Leite MI, Nakashima I, Waters P, McNeillis B, Brown R, et al. Prognostic factors and disease course in aquaporin-4 antibody-positive patients with neuromyelitis optica spectrum disorder from the United Kingdom and Japan. Brain. 2012;135:1834-49. 


\section{Figure Legend}

Figure 1. Quality of life (SF36) and pain severity (PSI) are significantly correlated in both the AQP4-Ab +ve group $(p=0.000007)$ and AQP4-Ab -ve group $(p=0.002)$. The presence of bilateral visual impairment from optic neuritis (ON, black cross) did not appear to affect this correlation in the AQP4-Ab +ve group. 
Table 1. Clinical profiles in NMO patients

\begin{tabular}{|c|c|c|c|c|}
\hline & Total & AQP4ab +ve & AQP4ab -ve & Mann-Whitney (p) \\
\hline $\mathrm{N}$ & 43 & 29 & 14 & \\
\hline Median age years (range) & & $52.5(23-76)$ & $56(28-77)$ & 0.547 \\
\hline Female : male & & $25 \mathrm{~F} 4 \mathrm{M}$ & $10 \mathrm{~F} 4 \mathrm{M}$ & 0.51 \\
\hline $\begin{array}{c}\text { Ethnicity(Caucasian, Asian, } \\
\text { Afro-Caribbean) }\end{array}$ & & $22 \mathrm{C} / 4 \mathrm{~A} / 3 \mathrm{AC}$ & $11 \mathrm{C} / 1 \mathrm{~A} / 2 \mathrm{AC}$ & $\overline{N A}$ \\
\hline LETM: short lesion TM & & $20: 9$ & $9: 5$ & 0.685 \\
\hline $\begin{array}{l}\text { Significant bilateral visual } \\
\text { impairment }(n)\end{array}$ & & 4 & 1 & \\
\hline PSI & & $20(0-38)$ & $15(0-34)$ & 0.162 \\
\hline PCS & & $15(0-48)$ & $10(0-37)$ & 0.528 \\
\hline HADS & & $14(0-38)$ & $13.5(5-39)$ & 0.834 \\
\hline SF-36 & & $1412.5(65-3400)$ & $1407(455-3030)$ & 0.944 \\
\hline EDMUS & & $5(1-8)$ & $3.5(1-8)$ & 0.347 \\
\hline Lesion length & & $7(0-16)$ & $6.1(1-31)$ & 0.664 \\
\hline Relapses & & $3(1-11)$ & $2.5(1-11)$ & 0.276 \\
\hline
\end{tabular}


Table 2. Correlation tests between pain and other measures

\begin{tabular}{|c|l|l|l|}
\hline PSI correlation $\mathbf{r}(\mathbf{p})$ & PCS & HADS & EDMUS \\
(Controlled by gender, age, lesion type) & & & \\
\hline Total group & $0.51(0.001)$ & $0.48(0.003)$ & $0.41(0.013)$ \\
\hline AQP4ab +ve & $0.38(0.074)$ & $0.38(0.074)$ & $0.45(0.03)$ \\
\hline AQP4ab -ve & $0.81(0.003)$ & $0.78(0.005)$ & $0.20(0.50)$ \\
\hline Comparison between groups (p) & $\mathbf{0 . 0 5}$ & 0.11 & 0.47 \\
\hline \multicolumn{2}{|l|}{} & & \\
\hline
\end{tabular}


Table 3. Linear regression test for all factors as predictor of SF36

\begin{tabular}{|c|c|c|c|c|c|c|}
\hline \multirow{2}{*}{ Factors } & \multicolumn{2}{|c|}{ Total group } & \multicolumn{2}{|c|}{ AQP4 ab +ve } & \multicolumn{2}{|c|}{ AQP4 ab -ve } \\
\hline & $\mathbf{p}$ & Beta & $p$ & Beta & p & Beta \\
\hline PSI & 0.0002 & -0.417 & 0.00035 & -0.435 & 0.308 & -0.342 \\
\hline Age & 0.001 & -0.304 & 0.186 & -0.129 & 0.036 & -0.512 \\
\hline HADS & 0.066 & -0.236 & 0.018 & -0.330 & 0.943 & 0.028 \\
\hline EDMUS & 0.113 & -0.150 & 0.015 & -0.270 & 0.935 & -0.018 \\
\hline PCS & 0.163 & -0.182 & 0.378 & -0.114 & 0.197 & -0.558 \\
\hline
\end{tabular}

Beta: standardised coefficients 
Table s1. Full correlation tests for all measures in all groups

\begin{tabular}{|c|c|c|c|c|c|c|c|c|c|c|}
\hline $\mathrm{R}(\mathrm{p})$ & PSI & EDMUS & HADS & PCS & SF36 & GENDER & $\begin{array}{l}\text { LESION } \\
\text { TYPE }\end{array}$ & AGE & $\begin{array}{l}\text { LESION } \\
\text { LENGTH }\end{array}$ & $\begin{array}{l}\text { NUMBER OF } \\
\text { RELAPSES }\end{array}$ \\
\hline PSI & 1 & $\begin{array}{c}0.431 \\
(0.005)\end{array}$ & $\begin{array}{c}0.458 \\
(0.003)\end{array}$ & $\begin{array}{c}0.551 \\
(0.00023)\end{array}$ & $\begin{array}{c}-0.733 \\
\left(10^{-6}\right)\end{array}$ & $\begin{array}{c}0.022 \\
(0.894)\end{array}$ & $\begin{array}{l}-0.193 \\
(0.234)\end{array}$ & $\begin{array}{c}0.096 \\
(0.555)\end{array}$ & $\begin{array}{c}0.132 \\
(0.417)\end{array}$ & $\begin{array}{c}0.015 \\
(0.927)\end{array}$ \\
\hline EDMUS & & 1 & $\begin{array}{c}0.217 \\
(0.179)\end{array}$ & $\begin{array}{c}0.268 \\
(0.095)\end{array}$ & $\begin{array}{c}-0.519 \\
(0.001)\end{array}$ & $\begin{array}{c}-0.099 \\
(0.544)\end{array}$ & $\begin{array}{l}-0.254 \\
(0.113)\end{array}$ & $\begin{array}{c}0.351 \\
(0.026)\end{array}$ & $\begin{array}{c}0.209 \\
(0.195)\end{array}$ & $\begin{array}{c}-0.010 \\
(0.951)\end{array}$ \\
\hline HADS & & & 1 & $\begin{array}{l}0.756 \\
\left(10^{-6}\right)\end{array}$ & $\begin{array}{c}-0.659 \\
\left(10^{-6}\right)\end{array}$ & $\begin{array}{c}-0.050 \\
(0.973)\end{array}$ & $\begin{array}{l}-0.134 \\
(0.408)\end{array}$ & $\begin{array}{c}0.1 \\
(0.540)\end{array}$ & $\begin{array}{c}0.16 \\
(0.325)\end{array}$ & $\begin{array}{c}-0.047 \\
(0.772)\end{array}$ \\
\hline PCS & & & & 1 & $\begin{array}{c}-0.668 \\
\left(2 \times 10^{-6}\right)\end{array}$ & $\begin{array}{c}0.222 \\
(0.168)\end{array}$ & $\begin{array}{l}-0.184 \\
(0.257)\end{array}$ & $\begin{array}{c}-0.064 \\
(0.694)\end{array}$ & $\begin{array}{c}0.191 \\
(0.238)\end{array}$ & $\begin{array}{c}-0.088 \\
(0.589)\end{array}$ \\
\hline SF36 & & & & & 1 & $\begin{array}{c}0.054 \\
(0.740)\end{array}$ & $\begin{array}{c}0.119 \\
(0.466)\end{array}$ & $\begin{array}{l}-0.365 \\
(0.021)\end{array}$ & $\begin{array}{l}-0.183 \\
(0.26)\end{array}$ & $\begin{array}{c}0.018 \\
(0.912)\end{array}$ \\
\hline GENDER & & & & & & 1 & $\begin{array}{c}0 \\
(1)\end{array}$ & $\begin{array}{c}-0.390 \\
(0.013)\end{array}$ & $\begin{array}{c}0.31 \\
(0.052)\end{array}$ & $\begin{array}{c}0.118 \\
(0.468)\end{array}$ \\
\hline LESION & & & & & & & 1 & $\begin{array}{l}-0.166 \\
(0.307)\end{array}$ & \begin{tabular}{|c|}
0.447 \\
$(0.004)$
\end{tabular} & $\begin{array}{c}0.495 \\
(0.001)\end{array}$ \\
\hline AGE & & & & & & & & 1 & $\begin{array}{c}-0.027 \\
(0.870)\end{array}$ & $\begin{array}{c}-0.223 \\
(0.167)\end{array}$ \\
\hline $\begin{array}{l}\text { LESION } \\
\text { LENGTH }\end{array}$ & & & & & & & & & 1 & $\begin{array}{c}-0.074 \\
(0.648)\end{array}$ \\
\hline $\begin{array}{l}\text { NUMBER OF } \\
\text { RELAPSES }\end{array}$ & & & & & & & & & & 1 \\
\hline
\end{tabular}


Table s2. Full correlation tests for all measures in AQP4 ve+ group

\begin{tabular}{|c|c|c|c|c|c|c|c|c|c|c|}
\hline $\mathrm{R}(\mathrm{p})$ & PSI & EDMUS & HADS & PCS & SF36 & GENDER & LESION & AGE & $\begin{array}{c}\text { LESION } \\
\text { LENGTH }\end{array}$ & $\begin{array}{c}\text { NUMBER OF } \\
\text { RELAPSES }\end{array}$ \\
\hline PSI & 1 & $\begin{array}{c}0.466 \\
(0.016)\end{array}$ & $\begin{array}{c}0.357 \\
(0.073)\end{array}$ & $\begin{array}{c}0.466 \\
(0.016)\end{array}$ & $\begin{array}{c}-0.759 \\
\left(7 \times 10^{-6}\right)\end{array}$ & $\begin{array}{c}0.021 \\
(0.918)\end{array}$ & $\begin{array}{c}-0.265 \\
(0.191)\end{array}$ & $\begin{array}{c}0.256 \\
(0.208)\end{array}$ & $\begin{array}{c}0.240 \\
(0.239)\end{array}$ & $\begin{array}{c}-0.068 \\
(0.740)\end{array}$ \\
\hline EDMUS & & 1 & $\begin{array}{c}0.099 \\
(0.629)\end{array}$ & $\begin{array}{c}0.159 \\
(0.437)\end{array}$ & $\begin{array}{c}-0.56 \\
(0.003)\end{array}$ & $\begin{array}{c}-0.115 \\
(0.576)\end{array}$ & $\begin{array}{c}-0.24 \\
(0.237)\end{array}$ & $\begin{array}{c}0.507 \\
(0.008)\end{array}$ & $\begin{array}{c}0.231 \\
(0.255)\end{array}$ & $\begin{array}{c}0.004 \\
(0.984)\end{array}$ \\
\hline HADS & & & 1 & $\begin{array}{c}0.767 \\
\left(5 \times 10^{-6}\right)\end{array}$ & $\begin{array}{c}-0.62 \\
(0.001)\end{array}$ & $\begin{array}{c}-0.121 \\
(0.556)\end{array}$ & $\begin{array}{c}0.049 \\
(0.813)\end{array}$ & $\begin{array}{c}0.189 \\
(0.356)\end{array}$ & $\begin{array}{c}0.087 \\
(0.672)\end{array}$ & $\begin{array}{c}-0.001 \\
(0.997)\end{array}$ \\
\hline PCS & & & & 1 & $\begin{array}{c}-0.661 \\
(0.00024)\end{array}$ & $\begin{array}{c}0.213 \\
(0.295)\end{array}$ & $\begin{array}{c}-0.14 \\
(0.494)\end{array}$ & $\begin{array}{c}0.152 \\
(0.458)\end{array}$ & $\begin{array}{c}0.114 \\
(0.579)\end{array}$ & $\begin{array}{l}-0.203 \\
(0.321)\end{array}$ \\
\hline SF36 & & & & & 1 & $\begin{array}{c}0.114 \\
(0.580)\end{array}$ & $\begin{array}{c}0.113 \\
(0.582)\end{array}$ & $\begin{array}{l}-0.459 \\
(0.018)\end{array}$ & $\begin{array}{c}-0.317 \\
(0.115)\end{array}$ & $\begin{array}{c}0.050 \\
(0.807)\end{array}$ \\
\hline GENDER & & & & & & 1 & $\begin{array}{c}0.086 \\
(0.676)\end{array}$ & $\begin{array}{c}-0.306 \\
(0.129)\end{array}$ & $\begin{array}{c}0.229 \\
(0.262)\end{array}$ & $\begin{array}{c}0.022 \\
(0.917)\end{array}$ \\
\hline LESION & & & & & & & 1 & $\begin{array}{l}-0.259 \\
(0.202)\end{array}$ & $\begin{array}{c}0.357 \\
(0.073)\end{array}$ & $\begin{array}{c}0.436 \\
(0.026)\end{array}$ \\
\hline AGE & & & & & & & & 1 & $\begin{array}{c}0.363 \\
(0.068)\end{array}$ & $\begin{array}{c}-0.114 \\
(0.578)\end{array}$ \\
\hline $\begin{array}{l}\text { LESION } \\
\text { LENGTH }\end{array}$ & & & & & & & & & 1 & $\begin{array}{l}-0.107 \\
(0.601)\end{array}$ \\
\hline $\begin{array}{l}\text { NUMBER OF } \\
\text { RELAPSES }\end{array}$ & & & & & & & & & & 1 \\
\hline
\end{tabular}


Table s3. Full correlation tests for all measures in AQP4 ve- group

\begin{tabular}{|c|c|c|c|c|c|c|c|c|c|c|}
\hline $\mathrm{R}(\mathrm{p})$ & PSI & EDMUS & HADS & PCS & SF36 & GENDER & LESION & AGE & $\begin{array}{c}\text { LESION } \\
\text { LENGTH }\end{array}$ & $\begin{array}{c}\text { NUMBER OF } \\
\text { RELAPSES }\end{array}$ \\
\hline PSI & 1 & $\begin{array}{c}0.253 \\
(0.384)\end{array}$ & $\begin{array}{c}0.687 \\
(0.007)\end{array}$ & $\begin{array}{c}0.738 \\
(0.003)\end{array}$ & $\begin{array}{l}-0.761 \\
(0.002)\end{array}$ & $\begin{array}{c}0.039 \\
(0.894)\end{array}$ & $\begin{array}{l}-0.090 \\
(0.761)\end{array}$ & $\begin{array}{c}-0.119 \\
(0.685)\end{array}$ & $\begin{array}{c}-0.041 \\
(0.890)\end{array}$ & $\begin{array}{c}-0.168 \\
(0.567)\end{array}$ \\
\hline EDMUS & & 1 & $\begin{array}{c}0.438 \\
(0.118)\end{array}$ & $\begin{array}{c}0.415 \\
(0.140)\end{array}$ & $\begin{array}{c}-0.401 \\
(0.155)\end{array}$ & $\begin{array}{c}-0.201 \\
(0.491)\end{array}$ & $\begin{array}{l}-0.275 \\
(0.341)\end{array}$ & $\begin{array}{c}0.120 \\
(0.684)\end{array}$ & $\begin{array}{c}0.196 \\
(0.502)\end{array}$ & $\begin{array}{c}-0.093 \\
(0.752)\end{array}$ \\
\hline HADS & & & 1 & $\begin{array}{c}0.728 \\
(0.003)\end{array}$ & $\begin{array}{l}-0.730 \\
(0.003)\end{array}$ & $\begin{array}{c}0.196 \\
(0.501)\end{array}$ & $\begin{array}{c}-0.484 \\
(0.080)\end{array}$ & $\begin{array}{c}-0.073 \\
(0.805)\end{array}$ & $\begin{array}{c}0.390 \\
(0.168)\end{array}$ & $\begin{array}{c}-0.248 \\
(0.392)\end{array}$ \\
\hline PCS & & & & 1 & $\begin{array}{l}-0.655 \\
(0.011)\end{array}$ & $\begin{array}{c}0.275 \\
(0.340)\end{array}$ & $\begin{array}{c}-0.251 \\
(0.386)\end{array}$ & $\begin{array}{c}-0.501 \\
(0.068)\end{array}$ & $\begin{array}{c}0.427 \\
(0.127)\end{array}$ & $\begin{array}{c}0.047 \\
(0.874)\end{array}$ \\
\hline SF36 & & & & & 1 & $\begin{array}{c}-0.078 \\
(0.790)\end{array}$ & $\begin{array}{c}0.107 \\
(0.715)\end{array}$ & $\begin{array}{c}-0.169 \\
(0.563)\end{array}$ & $\begin{array}{c}0.066 \\
(0.822)\end{array}$ & $\begin{array}{c}-0.018 \\
(0.951)\end{array}$ \\
\hline GENDER & & & & & & 1 & $\begin{array}{c}-0.091 \\
(0.756)\end{array}$ & $\begin{array}{c}-0.510 \\
(0.062)\end{array}$ & $\begin{array}{c}0.394 \\
(0.163)\end{array}$ & $\begin{array}{c}0.182 \\
(0.534)\end{array}$ \\
\hline LESION & & & & & & & 1 & $\begin{array}{c}-0.036 \\
(0.903)\end{array}$ & $\begin{array}{c}0.611 \\
(0.020)\end{array}$ & $\begin{array}{c}0.701 \\
(0.005)\end{array}$ \\
\hline AGE & & & & & & & & 1 & $\begin{array}{c}-0.636 \\
(0.014)\end{array}$ & $\begin{array}{c}-0.383 \\
(0.177)\end{array}$ \\
\hline $\begin{array}{l}\text { LESION } \\
\text { LENGTH }\end{array}$ & & & & & & & & & 1 & $\begin{array}{c}-0.089 \\
(0.763)\end{array}$ \\
\hline $\begin{array}{l}\text { NUMBER OF } \\
\text { RELAPSES }\end{array}$ & & & & & & & & & & 1 \\
\hline
\end{tabular}




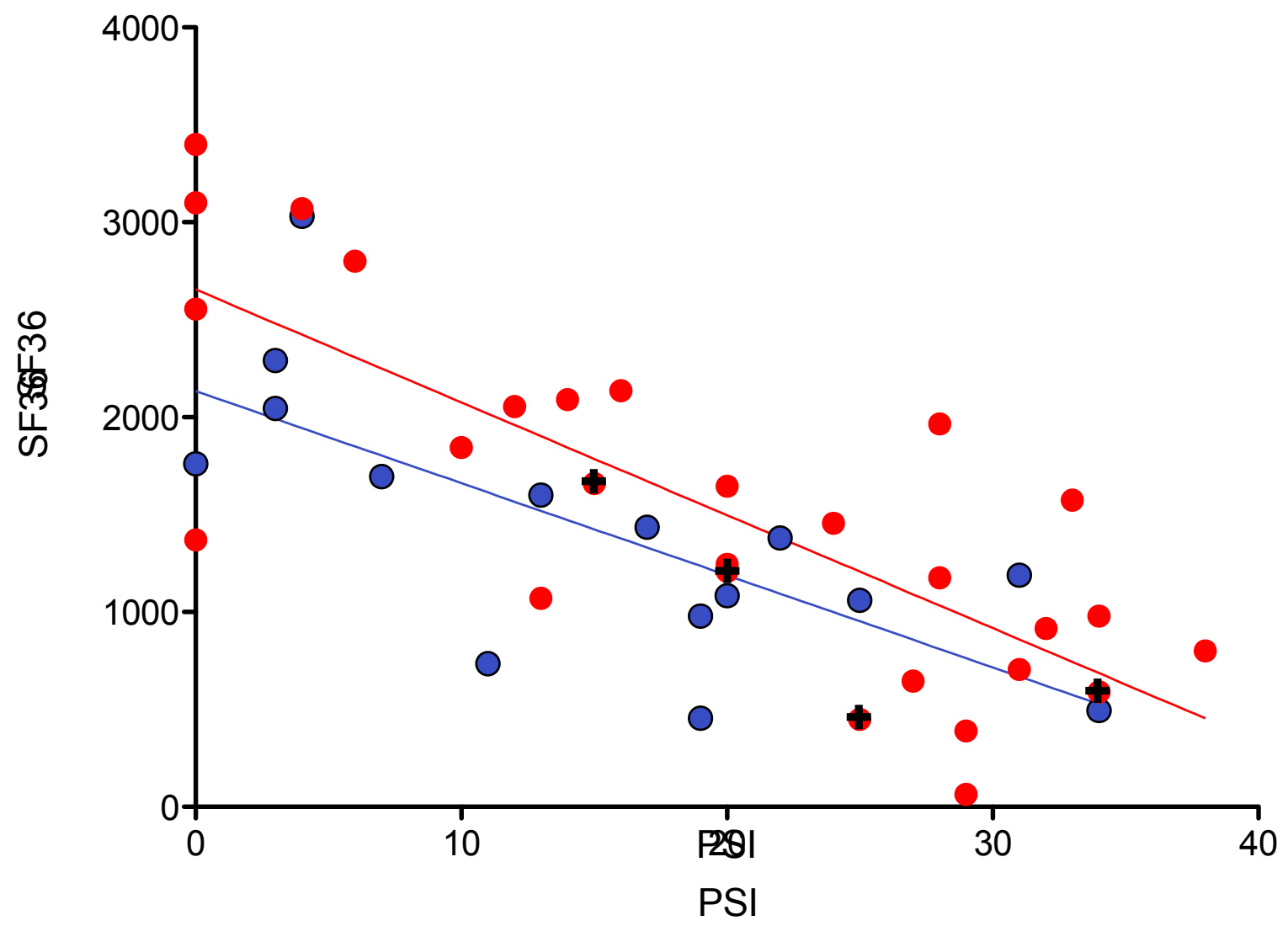

- AQP4-Abs +ve

- AQF4-Abs +ve ON

- - AQF4-Abs -ve 